\title{
The Determination of Antibiotic Resistance of Helicobacter Pylori Isolated from Patients Living in North of Iran (Sari)
}

\author{
Mojtaba Moradi Golrokhi ${ }^{1}$, Hafez Fakheri ${ }^{2}$, Mohammad Reza Haghshenas ${ }^{1}$, Mohammad Ahanjan ${ }^{3, *}$ \\ ${ }^{1}$ Microbiology Department, Faculty of Medicine, Mazandaran University of Medical Sciences, Iran \\ ${ }^{2}$ Internal Medicine Department, Inflammatory Diseases of Upper Gastrointestinal Tract Research Centre, Mazandaran University of \\ Medical Sciences, Iran \\ ${ }^{3}$ Department of Microbiology, Inflammatory Diseases of Upper Gastrointestinal Tract Research Center, \\ University of Medical Sciences, Iran
}

Copyright $(\mathcal{C} 2016$ by authors, all rights reserved. Authors agree that this article remains permanently open access under the terms of the Creative Commons Attribution License 4.0 International License

\begin{abstract}
Background: Helicobacter pylori (H. pylori), which infects about half of the world's population, is the main factor to peptic and duodenal ulcer diseases. Resistance to antibiotics is the main reason of failure of $H$. pylori treatment. Objective: Since the antibiotic resistance patterns of $H$. pylori is varied geographically; therefore, the main purpose of this study was to determine the antibacterial effects on $H$. pylori infection. Material and methods: The cross-sectional study was conducted on 120 patients (51 male, 69 female) with upper Gastrointestinal symptoms which they had referred to endoscopy department. Demographic characteristics of patients were recorded prior to sampling and the situations of resistance to 6 commonly used antibiotics in treating of $H$. pylori infection were studied. Results: In this study, 30 isolates were obtained from the culture of gastric biopsy specimens of 120 patients. The resistance rate of $H$. pylori to Metronidazole, Clarithromycin, Tetracycline, Amoxicillin and Levofloxacin were $63.3 \%, 16.6 \%, 6.6 \%, 10 \%$ and $3.3 \%$, respectively. There was no resistance rate of $\mathrm{H}$. pylori to Furazolidone during observation. Conclusion: According to antibiotic resistance level of this study, it seems that the antibiotic susceptibility tests are always necessary in order to determine the appropriate drug regimen against to $H$. pylori.
\end{abstract}

Keywords Antibiotic Resistance, Disk Diffusion, Helicobacter pylori, Iran

\section{Introduction}

Helicobacter pylori (H. pylori) is a gram-negative, microaerophilic, spiral with 4-6 flagellum on a pole which is approximately deployed in gastric of half the world's population (1). H. pylori is one of the most common infectious agents in humans, and the gastric is identified as most appropriate location for this bacterium. H. pylori can cause indigestion, gastric ulcer, duodenal ulcer and gastric cancer; it is transmitted through fecal-oral and oral-oral (2). There is a close relationship between $H$. pylori associated-infections with social, genetic and economic situations (3). $50-90 \%$ of populations are infected in developing countries and the infection often occurs in first years of life (3).

Antimicrobial treatment of $H$. pylori is difficult because it is located under gastric mucus layer. The rapid spread of drug resistance, reducing the effectiveness of certain medications and side effects of their uses are the factors which lead to dire needs to find new antibiotics without the drawbacks of pervious drug (4). Currently, the resistance of a bacterium to several antibacterial drugs has significantly increased. The getting of several irrelevant resistance gene and also, creation of mutations in a single gene or sets of genes is considered as the main reason for this event which is caused to resistance to a number of dissimilar compounds. Creation of multi-drug resistant strains by obtaining numerous genes occurs during successive stages of transgenesis and environmental selection in areas where the use of antimicrobial drugs is large (5). In some instances, this treatment regimen has not been successful; the development of resistance among strains of bacteria to antibiotics is one of reasons for failure (6).

Three mechanisms including changing the target, disabling of antibiotics by enzymes and prevention of accumulation of drugs into cells by efflux systems play significant roles in antibiotic resistance. Although a large number of drug resistance in bacteria is an inherent trait; but in many cases, this phenomenon is caused by the antibiotical pressure and is achieved by organisms (7). Therefore, the 
sensitivity and resistance level of bacteria to antibiotics should be measured in order to obtain the appropriate treatment formula (8). Since the pattern of $H$. pylori antibiotic resistance changes with respecting the geography, there is a serious need for regional studies to determine and prescribe appropriate medications (9). The antibiotic resistance of $H$. pylori is increasing in Iran during recently decades (10). This is considered as major concern for communities. And also the rare recent information published on resistance of $H$. pylori to antibiotics in a large population in the north of Iran is more than previous decades. Therefore, the identification of $H$. pylori resistance pattern to antimicrobial agents is essential for suitable treatment of these bacterial infections. Finally, we assessed the prevalence of $H$. pylori resistance against six antibiotics (Metronidazole, Clarithromycin, Tetracycline, Amoxicillin, Furazolidone and Levofloxacin) among the obtained strains from gastric biopsies of patients referred to Imam Khomeini Hospital of Sari city (Iran) by diffusion disk.

\section{Material and Methods}

\section{Study population}

One hundred twenty patients (120), (51 of the patients were males (23-89 years old) and 69 of female patients (17-86 years old) presenting with upper gastrointestinal (GI) symptoms were studied. In this cross-sectional study we referred patients to endoscopy department of Imam Khomeini Hospital of Sari in north of Iran during the November 2013 to June 2014. We selected patients $(32(26.66 \%)$ of the patients were from rural region and $88(73.34 \%)$ of patients lived in city), which had not used GI drugs over the past months, due to a reduction in their chances of positive cultures. The demographic data including of: age, sex, symptoms, and medication was collected through the questionnaire and were analyzed. Sampling was done with the consent of the patient. Samples were taken by an experienced physician from the antral area gastric. Tow biopsy specimen of any person (the first sample were inoculated into the Rapid urease test (RUT) broth (Merck, Germany) and second sample were added into Stuart transitional medium (Merck, Germany) or the broth BHI (Merck, Germany)) were obtained. The samples were transferred to Research Laboratory of Microbiology of Medical School within 3 hours. The urease positive biopsies, which were in transitional medium, were completely crushed between 2 sterile glass slides and the obtained extract was cultured in medium containing $10 \%$ sheep blood and specific supplements containing antibiotics Vancomycin, Polymyxin and and Trimethoprim (Merck, Germany) in microaerophilic conditions at $37^{\circ} \mathrm{C}$ for $3-5$ days in an $\mathrm{CO}_{2}$ incubator (12). The obtained and suspected colonies of $H$. pylori were verified by observing of the morphology, gram stain, catalase and oxidase and biochemical tests were confirmed.

\section{Determination of antibiotic susceptibility}

To determine the antibiotical susceptibility pattern, the disk diffusion method was employed on Mueller Hinton agar (Merck, Germany) which had been enriched with defibrinated blood of sheep. As follow: at first, the bacterial suspension equivalent to $3 \mathrm{McFarland}$ was prepared and cultured in enriched medium of Mueller Hinton agar. Then the antibiotic disks were placed on the surface of agar. The discs were incubated for $72 \mathrm{~h}$ in microaerophilic conditions and the zone around the disks was measured and recorded by a ruler based on standards (National Committee for Clinical Laboratory Standards (NCCLS) (8). Results were recorded as resistance according to the following interpretive criteria: for Clarithromycin, a zone $\geq 1 \mu \mathrm{g} / \mathrm{mL}$; for Metronidazole a zone $\geq 8 \mu \mathrm{g} / \mathrm{mL}$; for Amoxicillin, a zone $\geq 0.5 \mu \mathrm{g} / \mathrm{mL}$. These break points were used based on the recommendations of the NCCLS.

\section{Statistical analysis}

Data were recorded into the environment of 16 SPSS statistical software (version 19, SPSS Inc, Chicago, IL) and were analyzed using descriptive statistics (frequency and mean). Chi-square tests were used to determine statistical significance for comparisons of Resistance to antibiotics.

\section{Results}

The results showed that 65 patients $(53.3 \%)$ were infected with $H$. pylori. Results of this study indicated that the positive and negative samples were $65(53.3 \%)$ and 55 (46.7\%), respectively. And also, $30 \mathrm{H}$. pylori isolate (25\%) were obtained from culture. A total of individuals who were enrolled in the study proved that there was no relationship between gender and H.pylori infection. The age of patients ranged from 17 to 89 years. H.pylori was more common in the older patients $p$-value $(0.012)$. There was a relationship between age and infection with H.pylori p-value (0.012). The number and percentage resistance to Metronidazole, Amoxicillin, Clarithromycin, Tetracycline and Levofloxacin was 19 (63.3\%), 3 (10\%), 5 (16.6\%), 2 (6.6\%), 1 (3.3\%), respectively. Also, there was no observed resistance to Furazolidone. Figure 1 shows the resistance level of isolated strain to 6 common antibiotics. Among the positive samples, maximum resistance is observed against Metronidazole which it is presented in Figure 1. 


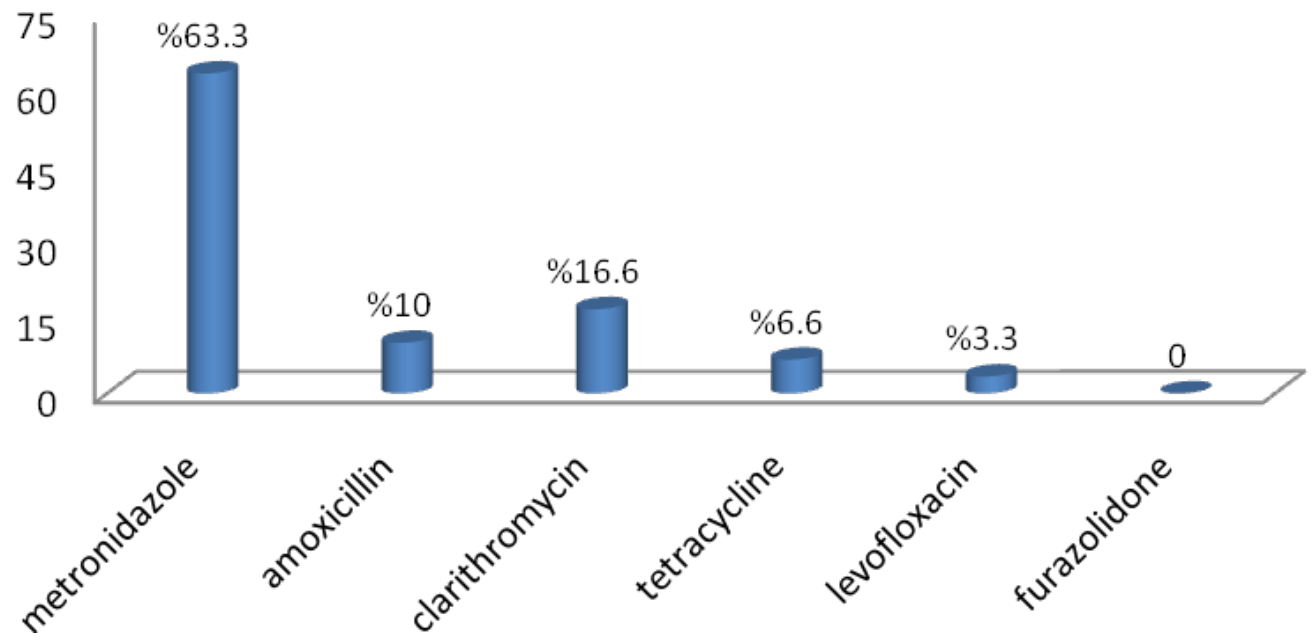

Figure 1. Distribution of among isolates of $H$. pylori resistance to antibiotics

\section{Discussion}

This study was conducted on $H$. pylori isolated from biopsies of patients with digestive problems referred to Imam Khomeini Hospital in the Sari city (Iran). The highest antibiotic resistance level was determined against Metronidazole (63.3\%), which was higher than pervious study in Iran (Mirzaei et al. and Mohammadi et al. have detected $56.3 \%$ and $57.5 \%$ resistance to this antibiotic, respectively), and the results of this study are close to their results $(9,10)$. And also antibiotic resistance level of Metronidazole was lower than the resistance level was reported $82.9 \%$ and $78.6 \%$ by Vilaichone et al. and Talebi et al. respectively in Bhutan and Iran, which indicated that higher resistance percentage compared with present work $(11,16)$. Antibiotic resistance patterns differ geographically and can change dramatically in relation to an increase in use of an antibiotic. It seems that the wide application of Metronidazole in H. pylori infections and other infections, such as parasitic infection, has been led to rise of resistance to these antibiotics. The extensive use of Metronidazole kills the susceptible isolates and helps to multiply the resistant and in minority strains (17).

Results of previous decades (Walsh et al. 1995; Debets-Ossenkopp et al. 1996) indicated that Clarithromycin was used in eradication therapy of $H$. pylori. Development of Clarithromycin resistance during therapy is a predominant cause of therapy reverse. The results of this study showed that the resistance of against Clarithromycin was obtained $16.6 \%$. The Islami et al. has implied that the resistance level against Clarithromycin in their study was $16.1 \%$ and $16 \%$, respectively, and their results can verify this study (13). However, the resistance level percentage to Clarithromycin in present work is inconsistent with other studies (13). It should be noted that the resistance against this antibiotic are increasing in Iran, so that the resistance percentage was $1.4 \%$ in 2006 (12). And also, it has been increased to $26.5 \%$ in 2013 in Iran (17). Clarithromycin belongs to macrolides antibiotics which were not used in past years due to high prices. Currently, this antibiotic is produced and widely used to treat the $H$. pylori infection which can be a reason for increasing of the resistance to this antibiotic. Also, the monotherapy use of this antibiotic can be another reason. The macrolides act by a similar mechanism and naturally, the bacteria become resistant with relatively similar mechanisms. Therefore, the widespread use of macrolide drugs to treat other infections may cause the resistance to Clarithromycin when it is used for $H$. pylori infection. It has been determined that the amount of macrolides Clarithromycin resistance in countries, which are widely used to treat infections, is higher than other countries with lesser use of this antibiotic (17). Different level of resistance is observed in other part of world. For example, Alarcon et al. has been reported that $23.9 \%$ resistance to this antibiotic (24).

In present work, Furazolidone was the most effective antibiotics against $H$. pylori because there was not detected resistance to it, but it should be noted that use of the drug has been banned in the United States by the food and drug administration in 1991 (34). Mohammed Savari and colleagues have not observed resistance to Furazolidone which is consistent with our study (18). $13 \%$ of resistance to this antibiotic was expressed in another study which is conducted by Paula Ortiz et al. (30). Siavoshi et al. also studied the Furazolidone resistance in Tehran (Iran) in 2010 and reported $4.5 \%$ of resistance (18). Meanwhile, because of the low cost and easily availability in developing countries of Furazolidone(7), this could be the beginning of the increasing of resistance to this antibiotic.

The resistance amount to Tetracycline was $6.6 \%$, which was lower than the results of Talebi et al. studies $(26,30)$ and higher than the results of Islami et al. (2013) and Vilaichone $(29,35)$. Shiota et al. there was no observed resistance rate of H. pylori to Tetracycline among male United States Veterans during 2015. Tetracycline resistance was rare in our study, which is consistent with previous studies $(19,20)$.

At the present work the resistance level to Amoxicillin was $10 \%$, that was similar with the results of Talebi et al and higher than the results of Khademi et al, which have been 
determined that resistance to Amoxicillin was 6.4\% (11). Farshad et al. has been found that the resistance rate to this antibiotic was $19 \%$ (18) which was higher than the results of our study. Also, there was no observed resistance to this antibiotic in study of Alarcon et al. (24).

In the present work, the resistance level to Levofloxacin was 3.3\%, which is consistent with Vilaichone et al. in 2013. They have been reported that the resistance to this antibiotic was $2.7 \%$ (16). Also, Talebi and et al. have been observed that the resistance to this antibiotic was $5.3 \%$ (11) which is higher than the results of this study.

\section{Conclusions}

According to findings of our results, the maximum resistance level was observed against two antibiotics, Metronidazole and Clarithromycin, which indicates the necessity to use the proper alternatives in therapeutic regimen. The determination of drug resistance patterns of bacteria in different geographical regions of treatment will be necessary. It is strongly recommended that the physician should be significantly careful in selecting and prescribing of drugs. Also, the educational programs about the identification and use of antibiotics to patients should be done.

\section{REFERENCES}

[1] J. Uchiyama, H.Takeuchi, S-i. Kato , K. Gamoh , I. Takemura-Uchiyama, D. Ujihara T, Masanori, M. Shigenobu. Characterization of Helicobacter pylori Bacteriophage KHP30. American Society for Microbiology.Vol. 79,No.10, 3176-84. 2013.

[2] T. Loho, Diagnostic Tests for Helicobacter pylori Infection. The Indonesian Journal of Gastroenterology, Hepatology, and Digestive Endoscopy, Vol.9No.1,16-23, 2008.

[3] LM. Brown. Helicobacter pylori: epidemiology and routes of transmission. Epidemiol Rev.Vol.22, No.2, 283-97.2000.

[4] F. Siavoshi , F. Safari , D. Doratotaj , GR. Khatami , GH. Fallahi, MM. Mirnaseri . Antimicrobial resistance of Helicobacter pylori isolates from Iranian adults and children. Archives of Iranian medicine. Vol.9, No.4,308-14.2006.

[5] J. Kohanteb , A. Bazargani, M. Saberi-Firoozi, A. Mobasser . Antimicrobial susceptibility testing of Helicobacter pylori to selected agents by agar dilution method in Shiraz-Iran. Indian journal of medical microbiology. Vol.25, No.4,374-7.2007.

[6] F. Siavoshi ,P. Saniee , S. Latifi-Navid, S. Massarrat, A. Sheykholeslami . Increase in resistance rates of $\mathrm{H}$. pylori isolates to Metronidazole and Tetracycline--comparison of three 3-year studies. Archives of Iranian medicine. Vol.13, No.3,77-87.2010

[7] A.Talebi Bezmin Abadi, AM.Mobarez , T.Taghvaei , L.Wolfram . Antibiotic resistance of Helicobacter pylori in Mazandaran, North of Iran. Helicobacter. Vol.15, No.
$6,505-9.2010$

[8] National Committee for Clinical Laboratory Standards. Approved Standard M7-A5: Methods/or Dilution Antimicrobial Susceptibility Tests/or Bacteria That Grow Aerobically (National Committee for Clinical laboratory Standards, Wayne, PA) 2000.

[9] M.Mohammadi, D. Doroud, N. Mohajerani, S. Massarrat . Helicobacter pylori antibiotic resistance in Iran. World Journal of Gastroenterology. Vol. 11, No.38,6009-13.2005.

[10] N. Mirzaei , F.Poursina ,J. Faghri ,M. Talebi , MR. Khataminezhad,A. Hasanzadeh . Prevalence of Resistance of Helicobacter pylori Strains to Selected Antibiotics in Isfahan, Iran. Jundishapur Journal of Microbiology. Vol.3, Vol.6,No.5,1-6.2013.

[11] A. Talebi Bezmin Abadi, A. Ghasemzadeh, T.Taghvaei, AM. Mobarez. Primary resistance of Helicobacter pylori to Levofloxacin and moxifloxacine in Iran. J Clin Microbiol. Vol.46, No.5,1774-90.2008.

[12] S.Koletzko , F. Richy,P Bontems , J. Crone, N.Kalach, ML. Monteiro. Prospective multicenter study on antibiotic resistance of Helicobacter pylori strains obtained from children living in Europe. Gut.Vol. 55,No.12, 1711-6.2006.

[13] G.Eslami, S.Taheri,N. Baseri ,SA. Montazeri ,A. Shakeri,R. Samadie. Prevalence of Helicobacter pylori and Determination of Antibiotic Resistance in Patients with Gastritis Referred to Shahid Beheshti University of Medical Sciences Hospitals in Tehran Between 2010 and 2011. Arch Clin Infect Dis. Vol.8, No.1,18-22.2013.

[14] APO.Godoy ,Mi. Ribeiro,YHB. Benvengo ,1. Vitiello ,MDCB Miranda ,S. Mendonça . Analysis of antimicrobial susceptibility and virulence factors in Helicobacter pylori clinical isolates. BMC Gastroenterology. Vol.No.1-6.2003.

[15] V.De Francesco , E. Ierardi ,C. Hassan , A. Zullo . Furazolidone therapy for Helicobacter pylori: is it effective and safe? World J Gastroentrol.Vol. 15,No.15, 1914-5.

[16] R-K.Vilaichone ,P. Gumnarai, T. Ratanachu-ek,V. Mahachai . Nationwide survey of Helicobacter pylori antibiotic resistance in Thailand. Diagnostic Microbiology and Infectious Disease. Vol.77, 346-9.2013.

[17] F.Khademi ,J. Faghri ,F. Poursina , BN. Esfahani ,S. Moghim ,H. Fazeli . Resistance pattern of Helicobacter pylori strains to Clarithromycin, Metronidazole, and Amoxicillin in Isfahan, Iran. Journal of Research in Medical Sciences.39-43.2013.

[18] S.Farshad ,A. Alborzi ,A. Japoni ,R. Ranjbar , KH.Asl „P. Badiee . Antimicrobial susceptibility of Helicobacter pylori strains isolated from patients in Shiraz, Southern Iran. World Journal of Gastroenterology. Vol.16, No.45, 5746-51.2010.

[19] S. Seiji, R. Rita, A.Abeer, B.Hashem, Ei-Serag, Y.G.David. Antibiotic Resistance of Helicobacter pylori Among Male United States Veterans. Clinical Gastroenterology and Hepatology. Vol.13, 1616-1624.2015.

[20] JM. Meyer, NP.Silliman, W. Wang. Risk factors for Helicobacter pylori resistance in the United States: the surveillance of $\mathrm{H}$. pylori antimicrobial resistance partnership (SHARP) study, 1993-1999. Ann Intern Med, Vol.136,1324.2002.

[21] WM. Duck,J. Sobel ,JM. Pruckler . Antimicrobial resistance 
incidence and risk factors among Helicobacter pylori-infected persons, United States. Emerg Infect Dis.Vol.10,10881094.2004.

[22] JH. Walsh, WL. Peterson. The treatment of Helicobacter pylori infection in the management of peptic ulcer disease.N.Engl, Vol.12No.15, 984-91.1995.

[23] YJ. Debets-Ossenkopp, M. Sparrius, JG. Kusters, JJ.
Kolkman , CM. Vandenbroucke-Grauls. Mechanism of clarithromycin resistance in clinical isolates of Helicobacter pylori. FEMS Microbiol Lett. Vol. 15, No.142(1),37-42.199 6.

[24] T.Alarcon, D. Dominoo, M. Lopez-Brea. Antibiotic resistance problems with Helicobacter pylori. Int J Antimicrob Agents. Vol.12.no. 1. 19-26. 1999. 